\title{
NGScloud2: optimized bioinformatic analysis using Amazon Web Services
}

\author{
Fernando Mora-Márquez ${ }^{1}$, José Luis Vázquez-Poletti ${ }^{2}$, Unai López de Heredia ${ }^{\text {Corresp. } 1}$ \\ ${ }^{1}$ GI Sistemas Naturales e Historia Forestal, Dpto. Sistemas y Recursos Naturales, ETSI Montes, Forestal y del Medio Natural, Universidad Politécnica de \\ Madrid, Madrid, Spain \\ 2 GI Arquitectura de Sistemas Distribuidos, Dpto. de Arquitectura de Ordenadores y Automática, Facultad de Informática, Universidad Complutense de \\ Madrid, Madrid, Spain \\ Corresponding Author: Unai López de Heredia \\ Email address: unai.lopezdeheredia@upm.es
}

Background. NGScloud was a bioinformatic system developed to perform de novo RNAseq analysis of non-model species by exploiting the cloud computing capabilities of Amazon Web Services. The rapid changes undergone in the way this cloud computing service operates, along with the continuous release of novel bioinformatic applications to analyze next generation sequencing data, have made the software obsolete. NGScloud2 is an enhanced and expanded version of NGScloud that permits the access to ad hoc cloud computing infrastructure, scaled according to the complexity of each experiment.

Methods. NGScloud2 presents major technical improvements, such as the possibility of running spot instances and the most updated AWS instances types, that can lead to significant cost savings. As compared to its initial implementation, this improved version updates and includes common applications for de novo RNAseq analysis, and incorporates tools to operate workflows of bioinformatic analysis of reference-based RNAseq, RADseq and functional annotation. NGScloud2 optimizes the access to Amazon's large computing infrastructures to easily run popular bioinformatic software applications, otherwise inaccessible to non-specialized users lacking suitable hardware infrastructures.

Results. The correct performance of the pipelines for de novo RNAseq, reference-based RNAseq, RADseq and functional annotation was tested with real experimental data, providing tips to make optimal use of NGScloud2. Further, we provide a qualitative comparison of NGScloud2 vs. the Galaxy framework. NGScloud2 code, instructions for software installation and use are available at https://github.com/GGFHF/NGScloud2. NGScloud2 includes a companion package, NGShelper that contains python utilities to post-process the output of the pipelines for downstream analysis at https://github.com/GGFHF/NGShelper. 
1 NGScloud2: optimized bioinformatic analysis using

4 Amazon Web Services

Fernando Mora-Márquez ${ }^{1}$, José Luis Vázquez-Poletti ${ }^{2}$ and Unai López de Heredia ${ }^{1}$

${ }^{1}$ GI Sistemas Naturales e Historia Forestal, Dpto. Sistemas y Recursos Naturales, ETSI Montes, Forestal y del Medio Natural, Universidad Politécnica de Madrid, Madrid, Spain.

${ }^{2}$ GI Arquitectura de Sistemas Distribuidos, Dpto. de Arquitectura de Ordenadores y Automática, Facultad de Informática, Universidad Complutense de Madrid, Madrid, Spain.

Corresponding Author:

Unai López de Heredia ${ }^{1}$

C/ José Antonio Nováis 10, Madrid, 28040, Spain

Email address: unai.lopezdeheredia@upm.es

\section{Abstract}

Background. NGScloud was a bioinformatic system developed to perform de novo RNAseq analysis of non-model species by exploiting the cloud computing capabilities of Amazon Web Services. The rapid changes undergone in the way this cloud computing service operates, along with the continuous release of novel bioinformatic applications to analyze next generation sequencing data, have made the software obsolete. NGScloud2 is an enhanced and expanded version of NGScloud that permits the access to ad hoc cloud computing infrastructure, scaled according to the complexity of each experiment.

Methods. NGScloud2 presents major technical improvements, such as the possibility of running spot instances and the most updated AWS instances types, that can lead to significant cost savings. As compared to its initial implementation, this improved version updates and includes common applications for de novo RNAseq analysis, and incorporates tools to operate workflows of bioinformatic analysis of reference-based RNAseq, RADseq and functional annotation. NGScloud2 optimizes the access to Amazon's large computing infrastructures to easily run popular bioinformatic software applications, otherwise inaccessible to non-specialized users lacking suitable hardware infrastructures.

Results. The correct performance of the pipelines for de novo RNAseq, reference-based RNAseq, RADseq and functional annotation was tested with real experimental data, providing workflow performance estimates and tips to make optimal use of NGScloud2. Further, we provide a qualitative comparison of NGScloud2 vs. the Galaxy framework. NGScloud2 code, instructions for software installation and use are available at https://github.com/GGFHF/NGScloud2. NGScloud2 includes a companion package, NGShelper 
40 that contains python utilities to post-process the output of the pipelines for downstream analysis

41 at https://github.com/GGFHF/NGShelper.

42

\section{Introduction}

44 Next Generation Sequencing (NGS) has largely allowed the development of genomics and many fields of biological and life sciences (Frese et al., 2013). The way these experiments are conducted is not, however, trivial, and requires the use of suitable methods at all stages of the experiment to produce sound results. Moreover, NGS experiments must be dimensioned properly to be cost-efficient (Wordsworth et al., 2018).

A generic NGS experiment can be divided into two phases (López de Heredia, 2016). First, an in vitro phase that consists of the construction of genomic libraries (sets of nucleic acid fragments processed in the laboratory following the methodological instructions required by the sequencing technique used in the experiment) and sequencing of those libraries in an NGS platform to generate read files (see Heather \& Chain, 2016 for a description of the available sequencing platforms). Then, an in silico or bioinformatics phase is needed to process read sequencing files output by sequencing platforms. The bioinformatic analysis can be subdivided into three stages:(1) pre-processing of read files; (2) read assembly or read mapping; and (3) postprocessing of assembly/mapping results. In the preprocessing, the quality of the raw reads (those generated by the NGS platform without any modification) is evaluated. The causes that may produce a decrease in read quality and introduce bias in further inference, such as the presence of adapters and other sequences used in the construction of the libraries, bases with poor quality, PCR duplicates and possible experimental artifacts, etc., are eliminated. When there is not a reference genome or transcriptome, the preprocessed reads are assembled into larger fragments, or clustered, reconstructing the original DNA chains, originating contigs (continuous sequences obtained from the superposition of multiple reads) and scaffolds (ordered distribution of contigs that is inferred when paired reads are used maintaining gaps between them). When the reference genome/transcriptome exists, the preprocessed reads are mapped to the genome to determine the exact genomic region where they align. The post-processing stage will depend on the specific methodology employed in the analysis and on the aims of the experiments. Frequently, this stage consists of the assessment of the quality of the assembly/mapping, on the application of subsequent filters to find significant genomic variants or changes in expressional patterns, and on a functional annotation step to determine the biochemical and biological function of the postprocessed sequences.

The large output size of NGS technologies and the algorithms and applications employed in their analysis, present processing limitations typical of big data, such as RAM size, CPU capacity, storage and data accessibility (Yang et al., 2017). Therefore, research labs have to allocate a significant part of their budget to provisioning, managing and maintaining their computational infrastructure (Kwon et al., 2015). 
79 A cost-efficient alternative for bioinformatic analysis of NGS data that presents several

80 advantages over local or HPC hardware infrastructure resides in cloud computing (Langmead \&

81 Nellore, 2018), and several applications and platforms are optimized to operate bioinformatics on

82 cloud systems, such as the Galaxy framework (Afgan et al., 2018). Cloud computing is flexible

83 and scalable, allowing various configurations of OS, RAM size, CPU number and almost

84 unlimited storage to fit the hardware resources for a specific bioinformatics workflow. Once the

85 workflow computing requirements are provisioned, hardware resources are readily available, and

86 the workflow performance and data can be securely accessed and monitored at any time from

87 any local computer with internet access. Moreover, for public cloud services, the user only pays

88 for the effectively used resources, reducing experiment times and costs.

89 Amazon Web Services (AWS) is a public cloud computing platform that has a large number of

90 information technology infrastructure services. The Elastic Compute Cloud (EC2) is the AWS

91 service that provides computing capacity adjustable to the NGS experiment requirements with a

92 wide range of instances, currently more than 350 models with specific processors, number of

93 CPUs, RAM quantity and network type (https://aws.amazon.com/ec2/). EC2 instances (virtual

94 machines) can be grouped into: (1) general purpose instances (types t2, t3, t3a, m3, m4, m5 and

$95 \mathrm{~m} 5 \mathrm{a}$, among others), with a balanced mix of hardware resources; (2) compute optimized

96 instances (types c3, c4, c5, and c5a, among others), with high-performance processors and a

97 higher ratio of CPUs per RAM memory than instances for general use; and (3) memory

98 optimized instances (types r3, r4, r5 and r5a, among others), indicated for workloads with high

99 volume of data and with higher ratio of RAM memory per number of CPUs than general purpose

100 instances. A general-purpose instance typically has $4 \mathrm{GiB}$ of RAM per CPU; a compute

101 optimized instance, $2 \mathrm{GiB}$ per CPU; and a memory optimized instance, $8 \mathrm{GiB}$ per CPU. In

102 addition to the previous groups, there are accelerated computation instances that have hardware

103 accelerators or coprocessors and optimized storage used for large volumes of data. The

104 purchasing option of an instance can be on-demand, with a pay-per-use model that has a fixed

105 hourly price (https://aws.amazon.com/ec2/pricing/on-demand/); or spot, when the non-used EC2

106 computing capacity is requested. The spot instances have large discounts compared to the on-

107 demand hourly pricing (https://aws.amazon.com/ec2/spot/pricing) but, they have the advantage

108 that the instance can be stopped by AWS when it is required. The Elastic Block Store (EBS) is

109 the AWS storage service designed to store data from the EC2 service in named data volumes

110 (https://aws.amazon.com/ebs/), and has a fixed monthly quote per GiB.

111 Here we present NGScloud2, a new version of the NGScloud software (Mora-Márquez,

112 Vázquez-Poletti \& López de Heredia U, 2018). NGScloud was developed as a bioinformatics

113 system to perform de novo RNAseq analysis of non-model species. This was accomplished using

114 the cloud computing infrastructure from AWS (EC2) and its high-performance block storage

115 service (EBS). NGScloud allowed to create one or more EC2 instances (virtual machines) of m3,

116 c3 or $\mathrm{r} 3$ instance types forming clusters where analytic processes were run using StarCluster, an

117 open source cluster-computing toolkit for EC2 (http://star.mit.edu/cluster/). However, NGScloud

118 did not support the new instance types that AWS has made available since the original 
119 application release. Below we describe the major new features of NGScloud2 that significantly 120 expand NGScloud2 functionality with respect to the original version, providing workflows for

121 reference-based RNAseq, Restriction site Associated DNA sequencing (RADseq) and functional 122 annotation.

123

\section{4}

125

126

127

128

129

130

131

132

133

134

135

136

137

138

139

140

141

142

143

144

145

146

147

148

149

150

151

152

153

154

155

156

157

158

\section{Materials \& Methods}

NGScloud2 is a free and open source program written in Python3. Source code and a complete manual with installation instructions and tutorials to exploit all the potential of NGScloud2 are available from the GitHub repository (https://github.com/GGFHF/NGScloud2). NGScloud2 presents remarkable differences with respect to NGScloud both in the way AWS resources are managed to better exploit all the potential of EC2 and EBS, but also by incorporating the possibility of running a more complete set of bioinformatics applications and pipelines for $d e$ novo RNAseq, reference-based RNAseq, Restriction site Associated DNA sequencing (RADseq) and functional annotation. In addition, a toolkit of Python programs useful to post-process the output of RNAseq and RADseq experiments is available in NGShelper (https://github.com/GGFHF/NGShelper).

\section{Technical improvements}

NGScloud2 introduces a more efficient architecture of instances and volumes than the original version (Figure1). While NGScloud used one volume for each type of existing datasets (applications, databases, references, reads and results), NGScloud2 offers the possibility of holding all dataset types in a unique volume, thus reducing the complexity in volume management. NGSCloud2 philosophy is based on the "cluster" concept. A cluster is a set of 1 to $n$ virtual machines with the same instance type. Each instance type has its hardware features: processor type, CPU number, memory amount, etc. (https://aws.amazon.com/ec2/instancetypes/).

NGScloud2 includes two cluster modes, StarCluster and native. The StarCluster mode uses StarCluster (http://star.mit.edu/cluster/), an open source cluster-computing toolkit for EC2, which implements clusters of up to 20 virtual machines, enabling faster analysis. The last version of StarCluster (0.95.6) dates from 2013 and can only use AWS's previous generation instance types, i.e. $\mathrm{m} 3, \mathrm{c} 3$ or $\mathrm{r} 3$. In NGScloud2, we provide a patch to enable using $\mathrm{m} 4, \mathrm{c} 4$ and $\mathrm{r} 4$ instance types in the StarCluster mode.

To reduce the dependency of NGScloud from StarCluster, which only allows to create clusters of previous generation instances, NGScloud2 has incorporated a "native" instance creation mode that sets a single virtual machine with any of the currently available on-demand EC2 instance types ( $\mathrm{m} 4, \mathrm{c} 4, \mathrm{r} 4, \mathrm{~m} 5, \mathrm{~m} 5 \mathrm{a}, \mathrm{c} 5, \mathrm{c} 5 \mathrm{a}, \mathrm{r} 5$ and $\mathrm{r} 5 \mathrm{a})$. The new generation instance types are slightly cheaper and their hardware improves over equivalent hardware from previous generations. Moreover, the new version enables launching "spot instances" that derive from unused EC2 capacity in the AWS cloud (https://aws.amazon.com/ec2/spot/). Spot instances have the advantage of being up to $50-80 \%$ cheaper than on-demand instances at the cost of suffering 
159

160

161

162

163

164

165

166

167

168

169

170

171

172

173

174

175

176

177

178

179

180

181

182

183

184

185

186

187

188

189

190

191

192

193

194

195

196

197

198

unpredictable interruption out of control of the user (see the main characteristics of spot instances at https://aws.amazon.com/ec2/spot/pricing/). Therefore, using spot instances is highly recommended for data transfer and for certain bioinformatics processes that run fast, process small volume input or include the possibility to be re-launched from the process interruption point.

NGScloud2 includes a user-friendly graphical front-end to operate the hardware resources, submit processes, and manage the data. The front-end includes a drop-down menu to configure AWS resources (clusters, nodes and volumes) and to install available bioinformatics software. Data transfer between the cloud and the local computer is operated through another drop-down menu. Additional menus are available to run de novo RNAseq, reference-based RNAseq, RADseq and functional annotation workflows, respectively. Log files of each executed process can be consulted in the "Logs" menu.

\section{New methods and applications available}

The other major improvements of NGScloud2 over NGScloud are related to the implementation of new bioinformatics pipelines and application tools (Table 1) that are automatically installed using Bioconda (Grüning et al., 2018), thus giving access to updated versions of the software without worrying about dependencies and software requirements. While the original purpose of NGScloud was to help in de novo RNAseq analysis, NGScloud2 includes pipelines and applications to perform reference-based RNAseq, RADseq and functional annotation. The implemented bioinformatics applications were selected through a search of the total number of citations in JCR publications in the Web of Science. Some other utilities are original software applications implemented by our research group.

\section{De novo RNAseq}

The original software was mainly focused on de novo assembly of RNAseq libraries using either Trinity, and included pre-processing of reads with FASTQC (Andrews, 2010), Trimmomatic (Bolger, Lohse \& Usadel, 2014) and three de novo RNAseq assemblers: Trinity (Haas et al., 2013), SOAPdenovo-Trans (Xie et al., 2014) and Trans-ABySS (Robertson et al., 2010).

NGScloud2 de novo RNAseq workflow has been improved (Figure 2) by including cutadapt (Martin, 2011) to perform read pre-processing, a new read alignment step with Bowtie2 (Langmead \& Salzberg, 2012) to map back the reads to the assembled transcriptome and software to quantify total counts of transcripts for further differential expression analysis: eXpress (Roberts \& Pachter, 2013) and Kallisto (Bray el al., 2016). Intensive processes, such as Trinity and SOAPdenovo-Trans transcriptome assemblers can now be re-launched from the point where the process interruption occurred, thus preventing unexpected malfunctioning of the cloud system or software bugs (Mora-Márquez et al. 2020). A variant calling step is also included to find SNPs or indels using SAMtools (Li et al. 2009), BEDtools (Quinlan \& Hall, 2010) and BCFtools (Danecek \& McCarthy, 2017). 
199

200

201

202

203

204

205

206

207

208

209

210

211

212

213

214

215

216

217

218

219

220

221

222

223

224

225

226

227

228

229

230

231

232

233

234

235

236

237

238

\section{Reference-based RNAseq}

In the last years, an increasing number of genomic and transcriptomic resources are available for many plant and animal species. Therefore, reference-based RNAseq is expected to become a usual practice not only for model species. NGScloud2 includes a workflow to accomplish read pre-processing, read alignment, reference-guided assembly, quantitation, differential expression and variant calling (Figure 3). Read pre-processing is done with the same tools as for de novo RNAseq (Trimmomatic and cutadapt). Read alignment to a reference genome assembly can be performed with Bowtie2, or with popular splice-aware aligners: Hisat2 (Kim et al., 2019), TopHat2 (Kim et al., 2013), STAR (Dobin et al., 2013) or GSNAP (Wu et al., 2016). Moreover, read alignment can also be run against a reference transcriptome using GMAP (Wu et al., 2016). After read alignment, a transcriptome can be assembled using Cufflinks-Cuffmerge (Trapnell et al., 2012). Reference-guided de novo assembly can also be performed with Trinity's genome guided version (Haas et al., 2013). Transcript or isoform abundance can be quantified with Cuffquant (Trapnell et al., 2012) or HT-seq-count (Anders, Pyl \& Huber, 2015), and differential expression analysis can be run with Cuffdiff and Cuffnorm (Trapnell et al., 2012), or the expression matrix can be downloaded to run locally more up-to-date differential expression packages, such as DeSeq2 (Love et al., 2014) or edgeR (Robinson et al., 2010). A variant calling step that operates in a similar way than for de novo RNAseq is also included.

\section{$\underline{\text { RADseq }}$}

Another major novelty in NGScloud2 is the possibility of running RADseq bioinformatics workflows. This reduced genome representation methodology and its derivates (e.g. ddRADseq) are used to find out polymorphism in specific genomic regions nearby restriction enzyme cut sites in populations of multiple individuals, and has revealed powerful in phylogenetics, population genetics, and association mapping studies, among others (Andrews et al., 2016). In NGScloud2, we have included ddRADseqTools (Mora-Márquez et al., 2017) and RADdesigner (Guillardín-Calvo et al., 2019) to assess the optimal experimental design of a RADseq experiment, i.e. to choose the enzyme combinations, simulate the effect of allele dropout and PCR duplicates on coverage, quantify genotyping errors, optimize polymorphism detection parameters or determine sequencing depth coverage.

The workflow of RADseq data in NGScloud2 allows to analyze the data using two strategies (Figure 4). RADseq libraries can be mapped with Bowtie2, GSNAP or HISAT2 to an available genome or psedudogenome assembly. The pseudogenome can be assembled using the same (or complementary) reads with SOAPdenovo2 genomic assembler (Luo et al., 2012), or with the Starcode sequence clusterizer (Zorita, Cuscó \& Filion, 2015). After read mapping, variant calling is performed in a similar way than for de novo RNAseq. The alternative is to perform read clusterization, filtering and variant calling in a single step with the robust iPyrad pipeline (Eaton \& Overcast, 2020).

\section{Functional annotation}


239 As a last improvement over the original version, NGScloud2 encapsulates our standalone

240 application TOA (Mora-Márquez et al., 2021), so it can run in EC2. This application automates

241 the extraction of functional information from genomic databases, both plant specific (PLAZA)

242 and general-purpose genomic databases (NCBI's RefSeq and NR/NT), and the annotation of

243 sequences (Figure 5). TOA is a good complement for both RNAseq and ddRADseq workflows

244 in non-model plant species that has shown optimal performance in AWS's EC2 cloud. TOA aims

245 to establish workflows geared towards woody plant species that automate the extraction of

246 information from genomic databases and the annotation of sequences. TOA uses the following

247 databases: Dicots PLAZA 4.0, Monocots PLAZA 4.0, Gymno PLAZA 1.0, NCBI RefSeq Plant

248 and NCBI Nucleotide Database (NT) and NCBI Non-Redundant Protein Sequence Database

249 (NR). Although TOA was primarily designed to work with woody plant species, it can also be

250 used in the analysis of experiments on any type of plant organism. Additionally, NCBI Gene,

251 InterPro and Gene Ontology databases are also used to complete the information.

252

253 NGShelper

254 Besides the cloud infrastructure deployed in NGScloud2, we have included a companion

255 package, NGShelper that contains python utilities to post-process the output of NGScloud2

256 pipelines. The package contains some Bash (Linux) and Bat (Windows) scripts to facilitate

257 running the Python3 programs.

258 NGShelper facilitates format conversion of output files, filtering and subsetting of results, VCF

259 and FASTA files statistics extraction, among others. Utilities list and their usage and parameters

260 can be consulted at https://github.com/GGFHF/NGShelper/blob/master/Package/help.txt.

261

262

263

Validation of NGScloud2

264

The correct operability of the pipelines for de novo RNAseq, reference-based RNAseq, RADseq

265

and funcional annotation was tested with data generated by our research group. Test data for

266 RNAseq and RADseq workflows consisted of two sets of Illumina ${ }^{\mathrm{TM}}$ reads: (1) Pcan, a paired-

267 ended RNA library of xylem regeneration tissue of the conifer tree Pinus canariensis (Mora-

268 Márquez et al. 2020). (2) Suberintro, a set of 16 paired ended Illumina ${ }^{\mathrm{TM}}$ libraries of Quercus

269 suber, Quercus ilex and their hybrids obtained from leaf tissue; eight libraries correspond to genotyping-by-sequencing with MsII and other eight libraries correspond to ddRADseq with

270 PstI-MspI (see details in Guillardín-Calvo et al., 2019). Read data are available at NCBI:

271 SRX5228139-SRX5228161 for Pcan, and SRX5019123-SRX5019138 for Suberintro. The

272

273 functional annotation workflow was tested with a small subset of transcripts corresponding to the monolignol biosynthesis gene family in Arabidopsis (Raes et al., 2003).

274 We further compared the capabilities of NGScloud2 as compared to other bioinformatics

275 platforms that make use of cloud systems, such as the popular Galaxy framework (Afgan et al., 276 2018) or the iPlant Atmosphere service of Cyverse (Skidmore et al., 2011).

277

278

\section{Results}


280

281

282

283

284

285

286

287

288

289

290

291

292

293

294

295

296

297

298

299

300

301

302

303

304

305

306

307

308

309

310

311

312

313

314

315

316

317

318

319

320

\section{Validation of NGScloud2 operability}

We have checked the correct operability_of all the workflows and applications in NGScloud2 using real datasets and have estimated the performance of the most cost-efficient instance for each process in the workflows. The datasets including the files produced by the validation tests are available at Zenodo repositories (DOIs: 10.5281/zenodo.4554359, 10.5281/zenodo.4554621, and 10.5281/zenodo.4554857).

Data uploading, read quality assessment, trimming and preprocessing steps for RNAseq and RADseq workflows do not have excessive computational requirements (Tables 2, 3 and 4); therefore, they can be run efficiently using a cheap spot t3.medium instance (2 CPUs and $4 \mathrm{GiB}$ of RAM). Other tasks, however, need the deployment of more powerful instances. For instance, a memory-oriented r5.2xlarge instance ( 8 CPUs and $64 \mathrm{GiB}$ of RAM), has been used in the three transcriptome assembly processes tested in the de novo RNAseq assembly (Table 2).

For the reference-based RNAseq workflow, 23 PE libraries of an RNAseq experiment have been used in the tests, being the data volume much higher than in the de novo RNAseq workflow test. In this case, the most limiting step of the workflow consisted of read and transcriptome alignments performed with HISAT2 and GMAP, respectively (Table 3). These processes required an on-demand memory optimized r5.8xlarge instance (32 CPUs and $256 \mathrm{GiB}$ of RAM), which is four times the instance employed for de novo RNAseq assemblies, to reduce the times needed to complete the alignments. For RADseq data, which consisted of eight small SE libraries, an on-demand memory oriented instance r5.2xlarge (8 CPUs and 64 GiB of RAM) was used in the read alignment with Bowtie2 and GSNAP (Table 4).

For functional annotation, TOA configuration and the external genomic database upload do not have large hardware requirements (Table 5). Therefore, a t3.medium instance type (2 CPUs and $4 \mathrm{GiB}$ of RAM) was used, except for the database building processes of Refseq Plant proteome and NT and NR databases for BLAST+ and DIAMOND usage that have been run in a spot compute oriented c5.xlarge instance (4 CPUs and $8 \mathrm{GiB}$ of RAM) and a spot memory-oriented r5.xlarge (4CPUs and $32 \mathrm{GiB}$ of RAM). However, attention must be paid to the provided storage when dimensioning the experiment, because the internal TOA.db and intermediate files can reach $>1.5 \mathrm{~TB}$, mainly because of the size of the NCBI's NR/NT databases.

TOA annotation pipelines that use BLAST+ or DIAMOND were run in a spot r5.xlarge (4 CPUs and $32 \mathrm{GiB}$ ) instance because the test data is a small dataset (Table 6), but larger instance types are required to make times shorter.

\section{Comparison with other cloud platforms for bioinformatics}

Since Cyverse's iPlant Atmosphere service has restricted access, and is currently only available to researchers based in the US, we have only performed a qualitative comparison of NGScloud2 and Galaxy (Table 7) regarding expenses, availability, hardware characteristics and other factors that may affect cost-efficiency. From the comparison between the characteristics of both platforms, it appears that they are complementary. Galaxy is an open access platform that allows using a wide set of bioinformatics software tools to define and run user-defined workflows for NGS data analysis (genome assembly, RNAseq, etc.) on small or medium volume datasets. By

Peer) reviewing PDF | (2020:11:55904:1:1:NEW 5 Mar 2021) 
321

322

323

324

325

326

327

328

329

330

331

332

333

334

335

336

337

338

339

340

341

342

343

344

345

346

347

348

349

350

351

352

353

354

355

356

357

358

359

360

now, NGScloud2 has implemented de novo RNAseq, reference-based RNAseq, RADseq and functional annotation specific workflows for which the researcher can choose in a flexible way which bioinformatics application or applications to use in each task.

One of the biggest disadvantages of Galaxy compared to NGScloud2 is its limited storage space (only $250 \mathrm{~GB}$ ) that precludes running large experiments in an efficient way. Galaxy presents also limitations in the maximum number of CPUs and RAM that can be assigned to each process, and on the maximum number of concurrent jobs. Further, the user has to wait some time before the Galaxy infrastructure is set, leading to potential delays in getting the results of the analyses. Among NGScloud2 advantages, it is worth mentioning that AWS's cloud storage is potentially infinite, and that hardware infrastructure deployment and termination are immediate, allowing running many instances in parallel. Therefore NGScloud2 can be used to securely analyze small, medium, large or very large data without waiting times or walltime limitations, always having in mind that it is a pay-per-use service.

\section{Discussion}

Bioinformatics is one of the fields that has benefitted from the development of cloud infrastructure. Many software applications contain instructions to be run on cloud systems (e.g. Eaton \& Overcast, 2020 or https://github.com/bcgsc/transabyss/blob/master/TUTORIAL.md\#16mpi-and-multi-threading), but, very often, they require advanced knowledge about cloud infrastructure and how to configure it. Some other integral platforms are more similar to NGScloud2 philosophy, and aim to facilitate cloud instance deployment and data handling, at the same time they offer relatively easy access to software applications and workflow setting (e.g. iPlant Atmosphere and Galaxy). In comparison, NGScloud2 presents some advantages, mostly related to the almost unlimited storage and strong hardware infrastructure availability, the immediacy of the hardware infrastructure setup, and the easy configuration of applications and workflows. Unlike NGScloud2, although Galaxy has a wide range of bioinformatics tools, it does not currently implement applications for the design of RADseq experiments or for the comprehensive analysis of these type of data. Moreover, the informatic system developed in NGScloud2 is flexible enough to allow for further incorporation of new software applications in the future.

NGScloud2 has proved its operability for RNAseq, RADseq and functional annotation analysis, but it is important to select the optimal application for each workflow step according to the specific type of data to analyze (Conesa et al., 2016; López de Heredia \& Vázquez-Poletti, 2016) and the most cost-efficient instance to run each application (Mora-Márquez et al., 2018). Indeed, the instance type to use will depend on the hardware requirements of the bioinformatics software. NGScloud2 incorporates the possibility of using new generation $\mathrm{r} 5, \mathrm{c} 5$ and $\mathrm{m} 5$ instance types, that are more efficient and slightly cheaper than instances from previous generations.

Once the instance CPU and RAM are chosen, the purchasing option of the instance (spot or ondemand) will depend on the time the process will be in execution, which pivots usually on the data size and on the algorithm complexity. It must be taken into account that the longer time an

Peer) reviewing PDF | (2020:11:55904:1:1:NEW 5 Mar 2021) 
361

362

363

364

365

366

367

368

369

370

371

372

373

374

375

376

377

378

379

380

381

382

383

384

385

386

387

388

389

390

391

392

393

394

395

396

397

398

399

400

instance is running, the higher is the risk of being stopped. The current version of NGScloud2 allows restarting Trinity and SOAPdenovo-Trans processes from the point they stopped.

However, most of the applications in NGScloud2 are not designed to be restarted after an unexpected interruption, a common issue in spot instances. Therefore we recommend using ondemand instances in processes with long elapsed times, while other processes that do not require long runtimes or can be re-started after interruption, such as variant-calling, are preferably run on spot instances.

For RNAseq and RADseq workflows, data uploading and read preprocessing are easily done with low-power instances, therefore at a minimal cost. Transcriptome assembly and mapping to reference genome are the most limiting task of the workflows (Miller et al., 2010, Mora-Márquez et al., 2020), both in terms of the storage required for intermediate and output files and of the RAM and CPU number required to run the application. In these cases, memory oriented instances are more efficient than compute-oriented instances because the intrinsic nature of the assembly algorithms. It must be stressed out that memory and CPU requirements of de novo RNAseq assemblies grow linearly with read number for Trinity, and exponentially for SOAPdenovo-Trans (Mora-Márquez et al., 2020).

For functional annotation with TOA, the main hardware limitations are produced in the BLAST+ or DIAMOND homology search step that requires a minimum of $4 \mathrm{CPUs}$ and $32 \mathrm{GiB}$ instances for small datasets, but larger instance types are required for bigger datasets (e.g. a full transcriptome) or to reduce runtimes. It should be also noted that DIAMOND pipelines can be run in shorter times than BLAST+ pipelines (Buchfink et al., 2015; Mora-Márquez et al., 2021). A sufficient provision of storage volumes is also recommended to run the functional annotation workflow, to take full advantage of TOA capabilities.

\section{Conclusions}

NGScloud2 has significantly expanded the types of bioinformatics workflows to run using Amazon Web Services since its previous version. This new version has incorporated major technical improvements that optimize the use of popular software applications otherwise inaccessible to non-specialized users lacking suitable hardware infrastructures. Moreover, these technical improvements are oriented to significantly reduce costs by simplifying data access and taking advantage of EC2 spot instances that may produce savings of up to $50-80 \%$ in many steps of the analysis. Therefore, NGScloud2 constitutes a good alternative to other cloud-based platform to analyze RNAseq and RADseq data in model and non-model species.

\section{Acknowledgements}

We'd like to thank M. Hurtado and Dr. V.M. Chano for beta-testing of NGScloud2 operability.

\section{References}

Afgan, E., Baker, D., Batut, B., van den Beek, M., Bouvier, D., Čech, M., ... Blankenberg, D. (2018). The Galaxy platform for accessible, reproducible and collaborative biomedical 
401

402

403

404

405

406

407

408

409

410

411

412

413

414

415

416

417

418

419

420

421

422

423

424

425

426

427

428

429

430

431

432

433

434

435

436

437

438

439

analyses: 2018 update. Nucleic Acids Research, 46(W1), W537-W544. DOI:

10.1093/nar/gky379

Anders S, Pyl PT, Huber W. 2015. HTSeq--a Python framework to work with high-throughput sequencing data. Bioinformatics 31:166-169. DOI: 10.1093/bioinformatics/btu638.

Andrews KR, Good JM, Miller MR, Luikart G, Hohenlohe PA. 2016. Harnessing the power of RADseq for ecological and evolutionary genomics. Nature Reviews Genetics 17:81-92. DOI: $10.1038 / \mathrm{nrg} .2015 .28$.

Andrews S. 2010. FastQC: a quality control tool for high throughput sequence data. Available online at: http://www.bioinformatics.babraham.ac.uk/projects/fastqc.

Bolger AM, Lohse M, Usadel B. 2014. Trimmomatic: a flexible trimmer for Illumina sequence data. Bioinformatics 30:2114-2120. DOI: 10.1093/bioinformatics/btu170.

Bray NL, Pimentel H, Melsted P, Pachter L. 2016. Near-optimal probabilistic RNA-seq quantification. Nature Biotechnology 34:525-527. DOI: 10.1038/nbt.3519.

Buchfink, B., Xie, C., \& Huson, D. H. 2015. Fast and sensitive protein alignment using DIAMOND. Nature Methods, 12:59-60. DOI: 10.1038/nmeth.3176.

Bushmanova E, Antipov D, Lapidus A, Suvorov V, Prjibelski AD. 2016. rnaQUAST: a quality assessment tool for de novo transcriptome assemblies: Table 1. Bioinformatics 32:2210 2212. DOI: 10.1093/bioinformatics/btw218.

Camacho, C., Coulouris, G., Avagyan, V., Ma, N., Papadopoulos, J., Bealer, K., \& Madden, T. L. 2009. BLAST+: architecture and applications. BMC Bioinformatics, 10:421. DOI: 10.1186/1471-2105-10-421.

Conesa, A., Madrigal, P., Tarazona, S., Gomez-Cabrero, D., Cervera, A., McPherson, A., ... Mortazavi, A. 2016. A survey of best practices for RNA-seq data analysis. Genome Biology, 17:13. DOI: 10.1186/s13059-016-0881-8.

Danecek P, McCarthy SA. 2017. BCFtools/csq: haplotype-aware variant consequences. Bioinformatics 33:2037-2039. DOI: 10.1093/bioinformatics/btx100.

Dobin A, Davis CA, Schlesinger F, Drenkow J, Zaleski C, Jha S, Batut P, Chaisson M, Gingeras TR. 2013. STAR: ultrafast universal RNA-seq aligner. Bioinformatics 29:15-21. DOI: 10.1093/bioinformatics/bts635.

Eaton DAR, Overcast I. 2020. ipyrad: Interactive assembly and analysis of RADseq datasets. Bioinformatics 36:2592-2594. DOI: 10.1093/bioinformatics/btz966.

Frese KS, Katus HA, Meder B. 2013. Next-Generation Sequencing: From Understanding Biology to Personalized Medicine. Biology 2(1):378-398. DOI: 10.3390/biology2010378

Grüning B, Dale R, Sjödin A, Chapman BA, Rowe J, Tomkins-Tinch CH, Valieris R, Köster J. 2018. Bioconda: sustainable and comprehensive software distribution for the life sciences. Nature Methods 15:475-476. DOI: 10.1038/s41592-018-0046-7.

Guillardín-Calvo L, Mora-Márquez F, Soto Á, López de Heredia U. 2019. RADdesigner: a workflow to select the optimal sequencing methodology in genotyping experiments on woody plant species. Tree Genetics \& Genomes 15:64. DOI: 10.1007/s11295-019-1372-3. 
440 Gurevich A, Saveliev V, Vyahhi N, Tesler G. 2013. QUAST: quality assessment tool for genome

441

442

443

444

445

446

447

448

449

450

451

452

453

454

455

456

457

458

459

460

461

462

463

464

465

466

467

468

469

470

471

472

473

474

475

476

477

478 assemblies. Bioinformatics 29:1072-1075. DOI: 10.1093/bioinformatics/btt086.

Haas BJ, Papanicolaou A, Yassour M, Grabherr M, Blood PD, Bowden J, Couger MB, Eccles D, Li B, Lieber M, MacManes MD, Ott M, Orvis J, Pochet N, Strozzi F, Weeks N, Westerman R, William T, Dewey CN, Henschel R, LeDuc RD, Friedman N, Regev A. 2013. De novo transcript sequence reconstruction from RNA-seq using the Trinity platform for reference generation and analysis. Nature Protocols 8:1494-1512. DOI: 10.1038/nprot.2013.084.

Heather JM, Chain B. 2016. The sequence of sequencers: The history of sequencing DNA. Genomics 107(1):1-8. DOI: 10.1016/j.ygeno.2015.11.003.

Kim D, Paggi JM, Park C, Bennett C, Salzberg SL. 2019. Graph-based genome alignment and genotyping with HISAT2 and HISAT-genotype. Nature Biotechnology 37:907-915. DOI: 10.1038/s41587-019-0201-4.

Kim D, Pertea G, Trapnell C, Pimentel H, Kelley R, Salzberg SL. 2013. TopHat2: accurate alignment of transcriptomes in the presence of insertions, deletions and gene fusions. Genome Biology 14:R36. DOI: 10.1186/gb-2013-14-4-r36.

Kwon T, Yoo WG, Lee W-J, Kim W, Kim D-W. 2015. Next-generation sequencing data analysis on cloud computing. Genes \& Genomics 37:489-501. DOI: 10.1007/s13258-015-0280-7.

Langmead B, Nellore A. 2018. Cloud computing for genomic data analysis and collaboration. Nature Reviews Genetics 19:208-219. DOI: 10.1038/nrg.2017.113.

Langmead B, Salzberg SL. 2012. Fast gapped-read alignment with Bowtie 2. Nature Methods 9:357-359. DOI: 10.1038/nmeth.1923.

Li B, Fillmore N, Bai Y, Collins M, Thomson JA, Stewart R, Dewey CN. 2014. Evaluation of de novo transcriptome assemblies from RNA-Seq data. Genome Biology 15:553. DOI: 10.1186/s13059-014-0553-5.

Li H. 2011. Tabix: fast retrieval of sequence features from generic TAB-delimited files. Bioinformatics 27:718-719. DOI: 10.1093/bioinformatics/btq671.

Li H, Handsaker B, Wysoker A, Fennell T, Ruan J, Homer N, Marth G, Abecasis G, Durbin R. 2009. The Sequence Alignment/Map format and SAMtools. Bioinformatics 25:2078-2079. DOI: 10.1093/bioinformatics/btp352.

Li W, Godzik A. 2006. Cd-hit: a fast program for clustering and comparing large sets of protein or nucleotide sequences. Bioinformatics 22:1658-1659. DOI: 10.1093/bioinformatics/btl158.

López de Heredia, U. 2016. Las técnicas de secuenciación masiva en el estudio de la diversidad biológica. Munibe Ciencias Naturales, 64. DOI: 10.21630/mcn.2016.64.07

López de Heredia U, Vázquez-Poletti JL. 2016. RNA-seq analysis in forest tree species: bioinformatic problems and solutions. Tree Genetics \& Genomes 12:30 DOI:10.1007/s11295-016-0995-x.

Love MI, Huber W, Anders S. 2014. Moderated estimation of fold change and dispersion for RNA-seq data with DESeq2. Genome Biology 15: 550. DOI: 10.1186/s13059-014-0550-8. 
479

480

481

482

483

484

485

486

487

488

489

490

491

492

493

494

495

496

497

498

499

500

501

502

503

504

505

506

507

508

509

510

511

512

513

514

515

516

517

518

Luo R, Liu B, Xie Y, Li Z, Huang W, Yuan J, He G, Chen Y, Pan Q, Liu Y, Tang J, Wu G, Zhang H, Shi Y, Liu Y, Yu C, Wang B, Lu Y, Han C, Cheung DW, Yiu S-M, Peng S, Xiaoqian Z, Liu G, Liao X, Li Y, Yang H, Wang J, Lam T-W, Wang J. 2012. SOAPdenovo2: an empirically improved memory-efficient short-read de novo assembler. GigaScience 1:18. DOI: 10.1186/2047-217X-1-18.

Martin M. 2011. Cutadapt removes adapter sequences from high-throughput sequencing reads. EMBnet.journal 17:10-12. DOI: 10.14806/ej.17.1.200.

Miller, J. R., Koren, S., \& Sutton, G. 2010. Assembly algorithms for next-generation sequencing data. Genomics, 95(6), 315-327. DOI: 10.1016/j.ygeno.2010.03.001

Mora-Márquez, F., Chano, V., Vázquez-Poletti, J. L., \& López de Heredia, U. 2021. TOA: A software package for automated functional annotation in non-model plant species. Molecular Ecology Resources, 21(2), 621-636. DOI: 10.1111/1755-0998.13285.

Mora-Márquez F, García-Olivares V, Emerson BC, López de Heredia U. 2017. ddRADseqTools : a software package for in silico simulation and testing of double-digest RADseq experiments. Molecular Ecology Resources 17:230-246. DOI: 10.1111/17550998.12550.

Mora-Márquez F, Vázquez-Poletti JL, Chano V, Collada C, Soto Á, de Heredia UL. 2020. Hardware performance evaluation of de novo transcriptome assembly software in Amazon Elastic Compute Cloud. Current Bioinformatics 15:420-430. DOI: 10.2174/1574893615666191219095817.

Mora-Márquez F, Vázquez-Poletti JL, López de Heredia U. 2018. NGScloud: RNA-seq analysis of non-model species using cloud computing. Bioinformatics 34:3405-3407. DOI: 10.1093/bioinformatics/bty363.

Quinlan AR, Hall IM. 2010. BEDTools: a flexible suite of utilities for comparing genomic features. Bioinformatics 26:841-842. DOI: 10.1093/bioinformatics/btq033.

Raes J, Rohde A, Christensen JH, Van de Peer Y, Boerjan W. 2003. Genome-wide characterization of the lignification toolbox in Arabidopsis. Plant Physiology 133(3):10511071. DOI: $10.1104 / p p .103 .026484$

Roberts A, Pachter L. 2013. Streaming fragment assignment for real-time analysis of sequencing experiments. Nature Methods 10:71-73. DOI: 10.1038/nmeth.2251.

Robertson G, Schein J, Chiu R, Corbett R, Field M, Jackman SD, Mungall K, Lee S, Okada HM, Qian JQ, Griffith M, Raymond A, Thiessen N, Cezard T, Butterfield YS, Newsome R, Chan SK, She R, Varhol R, Kamoh B, Prabhu A-L, Tam A, Zhao Y, Moore RA, Hirst M, Marra MA, Jones SJM, Hoodless PA, Birol I. 2010. De novo assembly and analysis of RNA-seq data. Nature Methods 7:909-912. DOI: 10.1038/nmeth.1517.

Robinson MD, McCarthy DJ, Smyth GK, 2010. edgeR: a Bioconductor package for differential expression analysis of digital gene expression data. Bioinformatics 26(1):139-140. DOI: 10.1093/bioinformatics/btp616.

Skidmore, E., Kim, S., Kuchimanchi, S., Singaram, S., Merchant, N., \& Stanzione, D. (2011). iPlant atmosphere: A Gateway to Cloud Infrastructure for the Plant Sciences. Proceedings

PeerJ reviewing PDF | (2020:11:55904:1:1:NEW 5 Mar 2021) 
519

520

521

522

523

524

525

526

527

528

529

530

531

532

533

534

535

536

537

538

539

540

541

542

543

544

545

546

547

548

549

of the 2011 ACM Workshop on Gateway Computing Environments - GCE '11, 59. New York, New York, USA: ACM Press. DOI: 10.1145/2110486.2110495.

Smith-Unna R, Boursnell C, Patro R, Hibberd JM, Kelly S. 2016. TransRate: reference-free quality assessment of de novo transcriptome assemblies. Genome Research 26:1134-1144. DOI: $10.1101 /$ gr.196469.115.

Trapnell C, Roberts A, Goff L, Pertea G, Kim D, Kelley DR, Pimentel H, Salzberg SL, Rinn JL, Pachter L. 2012. Differential gene and transcript expression analysis of RNA-seq experiments with TopHat and Cufflinks. Nature Protocols 7:562-578. DOI: 10.1038/nprot.2012.016.

Waterhouse RM, Seppey M, Simão FA, Manni M, Ioannidis P, Klioutchnikov G, Kriventseva E V., Zdobnov EM. 2018. BUSCO Applications from Quality Assessments to Gene Prediction and Phylogenomics. Molecular Biology and Evolution 35:543-548. DOI: 10.1093/molbev/msx319.

Wordsworth S, Doble B, Payne K, Buchanan J, Marshall DA, McCabe C, Regier DA. 2018. Using "Big Data" in the Cost-Effectiveness Analysis of Next-Generation Sequencing Technologies: Challenges and Potential Solutions. Value Health 21(9):1048-1053. DOI: 10.1016/j.jval.2018.06.016.

Wu TD, Reeder J, Lawrence M, Becker G, Brauer MJ. 2016. GMAP and GSNAP for Genomic Sequence Alignment: Enhancements to Speed, Accuracy, and Functionality. In: Mathé E, Davis S eds. Statistical Genomics: Methods and Protocols. Springer Science+Business Media New York, 283-334. DOI: 10.1007/978-1-4939-3578-9_15.

Xie Y, Wu G, Tang J, Luo R, Patterson J, Liu S, Huang W, He G, Gu S, Li S, Zhou X, Lam TW, Li Y, Xu X, Wong GK-S, Wang J. 2014. SOAPdenovo-Trans: de novo transcriptome assembly with short RNA-Seq reads. Bioinformatics 30:1660-1666. DOI: 10.1093/bioinformatics/btu077.

Yang A, Troup M, Ho JWK. 2017. Scalability and Validation of Big Data Bioinformatics Software. Computational and Structural Biotechnology Journal 15:379-386. DOI: 10.1016/j.csbj.2017.07.002.

Zorita E, Cuscó P, Filion GJ. 2015. Starcode: sequence clustering based on all-pairs search. Bioinformatics 31:1913-1919. DOI: 10.1093/bioinformatics/btv053. 


\section{Table $\mathbf{1}$ (on next page)}

Software applications selected for de novo RNA-seq (dnRNAseq), reference-based RNAseq (rbRNAseq), RAD-seq (RADseq) and taxonomy-oriented annotation (TOA) workflows in NGScloud2.

t the number of citations were obtained according to the references listed in the Web of Science for each application 
1 Table 1. Software applications selected for de novo RNA-seq (dnRNAseq), reference-based RNA-seq (rbRNAseq),

2 RAD-seq (RADseq) and taxonomy-oriented annotation (TOA) workflows in NGScloud2.

3

\begin{tabular}{|c|c|c|c|c|}
\hline Software & Workflows & Task & Reference & $\#$ citations $\dagger$ \\
\hline BCFtools & dnRNAseq, rbRNAseq \& RADseq & variant calling & $\begin{array}{l}\text { Danecek \& McCarthy, } \\
2017\end{array}$ & 46 \\
\hline BEDtools & dnRNAseq, rbRNAseq \& RADseq & variant calling & Quinlan \& Hall, 2010 & 7531 \\
\hline Bowtie2 & dnRNAseq, rbRNAseq \& RADseq & read alignment & $\begin{array}{l}\text { Langmead \& Salzberg, } \\
2012\end{array}$ & 16309 \\
\hline BLAST+ & TOA & annotation pipeline & Camacho et al., 2009 & 5964 \\
\hline BUSCO & dnRNAseq & transcript quality & Waterhouse et al., 2018 & 584 \\
\hline $\begin{array}{l}\text { CD-HIT: } \\
\text { CD-HIT-EST }\end{array}$ & dnRNAseq & filtering & Li \& Godzik, 2006 & 4516 \\
\hline Cufflinks & rbRNAseq & $\begin{array}{l}\text { Cufflinks-Cuffmerge: } \\
\text { assembly } \\
\text { Cuffquant: quantitation } \\
\text { Cuffdiff: differential } \\
\text { expression }\end{array}$ & Trapnell et al., 2012 & 6679 \\
\hline cutadapt & dnRNAseq, rbRNAseq \& RADseq & preprocessisng & Martin, 2011 & 23 \\
\hline $\begin{array}{l}\text { DETONATE: } \\
\text { RSEM-EVAL }\end{array}$ & dnRNAseq & assembly quality & Li et al., 2014 & 125 \\
\hline ddRADseqTools & RADseq & experimental design & $\begin{array}{l}\text { Mora-Márquez et al., } \\
2017\end{array}$ & 11 \\
\hline DIAMOND & TOA & annotation pipeline & Buchfink et al., 2015 & 2083 \\
\hline eXpress & dnRNAseq & quantitation & Roberts \& Pachter, 2013 & 482 \\
\hline FastQC & dnRNAseq, rbRNAseq \& RADseq & preprocessisng & Andrews, 2010 & 127 \\
\hline
\end{tabular}

GMAP-GSNAP GMAP: rbRNAseq GMAP: transcriptome

$\begin{array}{ll}\text { GMAP-GSNAP } \quad \text { GSNAP: rbRNAseq \& RADseq } & \text { alignment } \\ \text { GSNAP: read alignment }\end{array}$

\begin{tabular}{|c|c|c|c|c|}
\hline HISAT2 & rbRNAseq & read alignment & Kim et al., 2019 & 288 \\
\hline $\begin{array}{l}\text { HTSeq: } \\
\text { ht-seq-count }\end{array}$ & rbRNAseq & quantitation & Anders et al., 2015 & 6906 \\
\hline ipyrad & RADseq & full pipeline & Eaton \& Overcast, 2020 & 30 \\
\hline Kallisto & dnRNAseq & quantitation & Bray et al., 2016 & 1883 \\
\hline NGShelper & dnRNAseq & $\begin{array}{l}\text { transcript-filtering: filtering } \\
\text { transcriptome-blast: } \\
\text { annotation }\end{array}$ & $\begin{array}{l}\text { https://github.com/GGFH } \\
\text { F/NGShelper }\end{array}$ & - \\
\hline QUAST & dnNRAseq & assembly quality & Gurevich et al., 2013 & 2061 \\
\hline RADdesigner & RADseq & design & $\begin{array}{l}\text { Guillardín-Calvo et al., } \\
2019\end{array}$ & 2 \\
\hline rnaQUAST & dnNRAseq & assembly quality & Bushmanova et al., 2016 & 31 \\
\hline SAMtools & dnRNAseq, rbRNAseq \& RADseq & variant calling & Li et al., 2009 & 4429 \\
\hline SOAPdenovo2 & RADseq & pseudo assembly & Luo et al., 2012 & 2558 \\
\hline $\begin{array}{l}\text { SOAPdenovo- } \\
\text { Trans }\end{array}$ & dnRNAseq & assembly & Xie et al., 2014 & 449 \\
\hline
\end{tabular}




\begin{tabular}{|c|c|c|c|c|}
\hline STAR & rbRNAseq & read alignment & Dobin et al., 2013 & 9718 \\
\hline Tabix & dnRNAseq, rbRNAseq \& RADseq & variant calling & $\mathrm{Li}, 2011$ & 195 \\
\hline TOA & TOA & all & $\begin{array}{l}\text { Mora-Márquez et al., } \\
2021\end{array}$ & - \\
\hline TopHat2 & rbRNAseq & read alignment & Kim et al., 2013 & 6912 \\
\hline Trans-ABySS & dnRNAseq & assembly & Robertson et al., 2010 & 542 \\
\hline TransDecoder & TOA & annotation pipeline & $\begin{array}{l}\text { https://github.com/Trans } \\
\text { Decoder }\end{array}$ & 45 \\
\hline Transrate & dnNRAseq & assembly quality & Smith-Unna et al., 2016 & 243 \\
\hline Trimmomatic & dnRNAseq, rbRNAseq \& RADseq & preprocessing & Bolger et al., 2014 & 14950 \\
\hline Trinity & $\begin{array}{l}\text { Trinity: dnRNAseq } \\
\text { genome-guided Trinity: rnRNAseq } \\
\text { insilico_read_normalization: } \\
\text { dnRNAseq }\end{array}$ & $\begin{array}{l}\text { Trinity: assembly } \\
\text { genome-guided Trinity: } \\
\text { assembly } \\
\text { insilico_read_normalization: } \\
\text { preprocessing }\end{array}$ & Haas et al., 2013 & 3330 \\
\hline
\end{tabular}

4 


\section{Table 2 (on next page)}

Tests of de novo RNA-seq workflow performance.

Output size and elapsed time (E.T.) consumed by each process and type of instance, number of CPUs and RAM employed to run each process. (*) Pcan1-S0_1.fastq.gz \& Pcan1SO_2.fastq.gz 
1 Table 2. Tests of de novo RNA-seq workflow performance. Output size and elapsed time (E.T.) consumed by each process and type of instance, number of

2 CPUs and RAM employed to run each process.

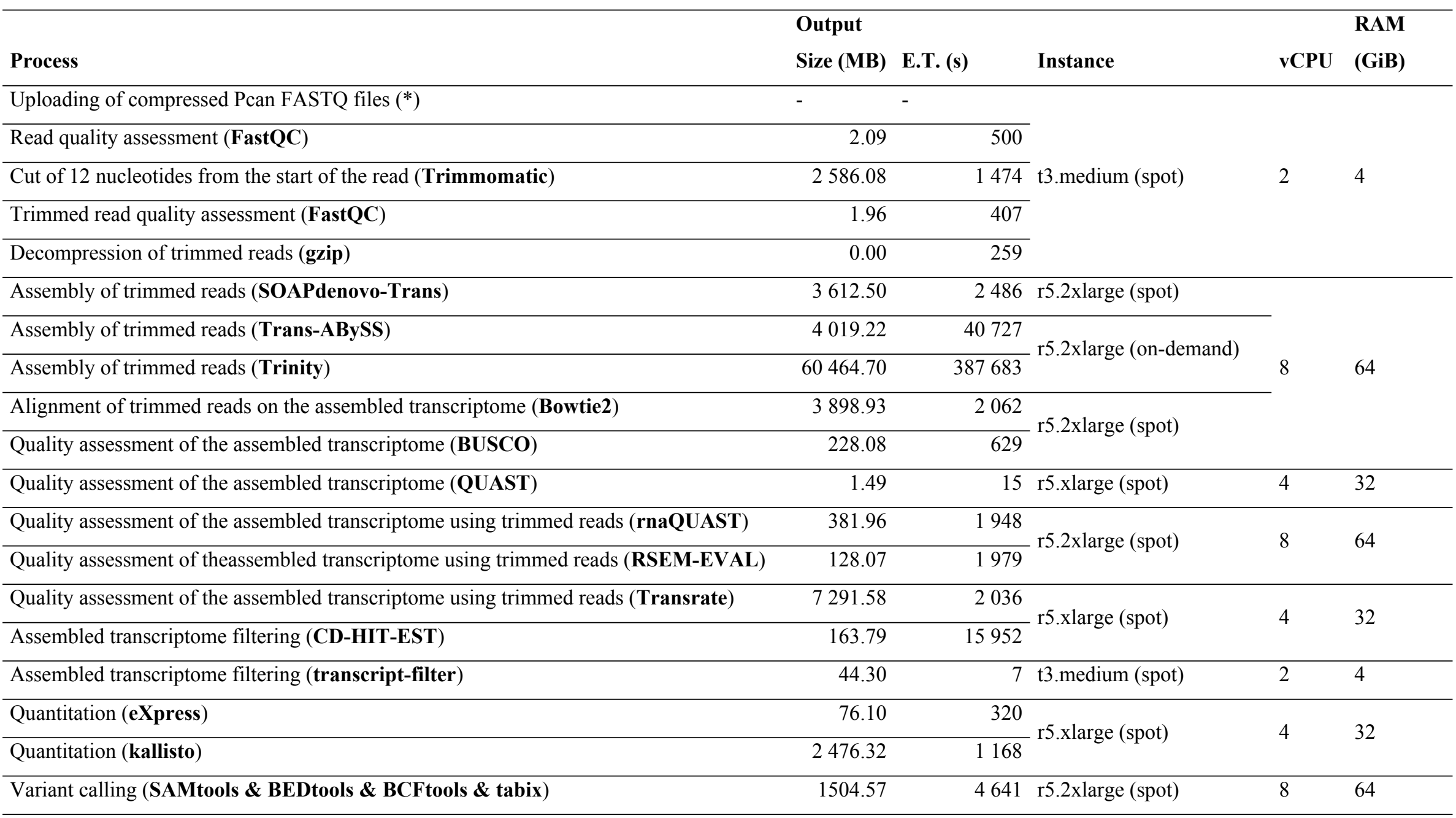

3 


\section{Table 3(on next page)}

Tests of reference-based RNA-seq workflow performance.

Output size and elapsed time (E.T.) consumed by each process and type of instance, number of CPUs and RAM employed to run each process. (*) all files correspond to the 23 libraries of Pcan 
1 Table 3. Tests of reference-based RNA-seq workflow performance. Output size and elapsed time (E.T.) consumed by each process and type of instance, number 2 of CPUs and RAM employed to run each process.

\begin{tabular}{|c|c|c|c|c|c|}
\hline Process & $\begin{array}{l}\text { Output } \\
\text { Size (MB) }\end{array}$ & E.T. (s) & Instance & vCPU & $\begin{array}{l}\text { RAM } \\
\text { (GiB) }\end{array}$ \\
\hline Uploading of compressed genome and annotation files of Pinus taeda & - & - & \multirow{7}{*}{ t3.medium (spot) } & \multirow{7}{*}{2} & \multirow{7}{*}{4} \\
\hline Decompression of genome and annotation files & 0.00 & 334 & & & \\
\hline Uploading of compressed Pcan FASTQ files $\left(^{*}\right)$ & - & - & & & \\
\hline Read quality assessment (FastQC) & \multirow{4}{*}{$\begin{array}{l}48.14 \\
-\quad 67037.63 \\
- \\
-45.21 \\
0.00\end{array}$} & \multirow{4}{*}{$\begin{array}{l}15642 \\
35758 \\
11631 \\
9169\end{array}$} & & & \\
\hline Cut of 12 nucleotides from the start of the read (Trimmomatic) & & & & & \\
\hline Trimmed read quality assessment (FastQC) & & & & & \\
\hline Decompression of trimmed reads (gzip) & & & & & \\
\hline Trimmed reads alignment to Pinus taeda genome (HISAT2) & 190994.33 & 73516 & $\begin{array}{l}\text { r5.8xlarge (on- } \\
\text { demand) }\end{array}$ & 32 & 256 \\
\hline Quantitation (htseq-count) & 3.26 & 9113 & m5.2xlarge & 8 & 32 \\
\hline Variant calling (SAMtools \& BEDtools \& BCFtools \& tabix) & 52041.37 & 60180 & (spot) & 8 & 32 \\
\hline Transcriptome alignment to Pinus taeda genome (GMAP) & 3046.34 & 35039 & $\begin{array}{l}\text { r5.8xlarge (on- } \\
\text { demand) }\end{array}$ & 32 & 256 \\
\hline
\end{tabular}

4 


\section{Table 4 (on next page)}

Tests of RAD-seq workflow performance.

Output size and elapsed time (E.T.) consumed by each process and type of instance, number of CPUs and RAM employed to run each process. (*) SRR8199746_2.fastq.gz, SRR8199747_2.fastq.gz, SRR8199748_2.fastq.gz, SRR8199749_2.fastq.gz, SRR8199750_2.fastq.gz, SRR8199751_2.fastq.gz, SRR8199760_2.fastq.gz \& SRR8199761_2.fastq.gz 
1 Table 4. Tests of RAD-seq workflow performance. Output size and elapsed time (E.T.) consumed by each process and type of instance, number of CPUs and

2 RAM employed to run each process.

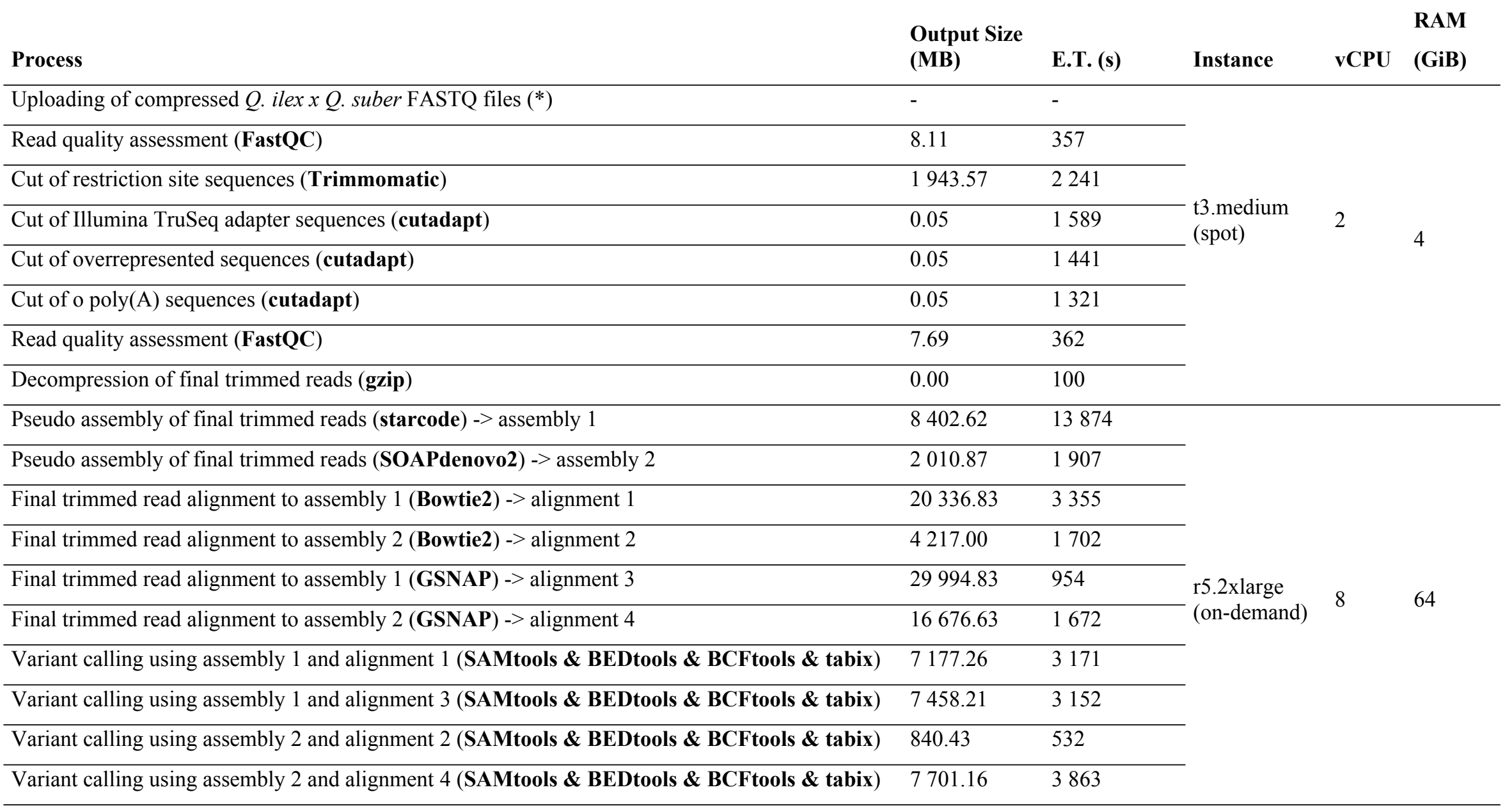




\section{Table 5 (on next page)}

Tests of TOA configuration and genomic database processes performance in the functional annotation workflow.

Output size and elapsed time (E.T.) consumed by each process and type of instance, number of CPUs and RAM employed to run each process. 
Table 5. Tests of TOA configuration and genomic database processes performance in the functional annotation workflow. Output size and elapsed

2 time (E.T.) consumed by each process and type of instance, number of CPUs and RAM employed to run each process.

\begin{tabular}{|c|c|c|c|c|c|}
\hline Process & $\begin{array}{l}\text { Output size } \\
\text { (MB) }\end{array}$ & E.T. (s) & Instance & vCPU & $\begin{array}{l}\text { RAM } \\
\text { (GiB) }\end{array}$ \\
\hline Creation of TOA config file & - & - & \multirow{15}{*}{$\begin{array}{l}\text { t3.medium } \\
\text { (spot) }\end{array}$} & \multirow{15}{*}{2} & \multirow{15}{*}{4} \\
\hline Creation of TOA database & 0.00 & 0 & & & \\
\hline Creation of genomic dataset file & - & - & & & \\
\hline Creation of species file & - & - & & & \\
\hline Download of other basic data & 0.02 & 10 & & & \\
\hline Load of basic data into TOA database & 0.02 & 57 & & & \\
\hline Build of Gymno PLAZA 1.0 proteome & 0.02 & 73 & & & \\
\hline Download of Gymno PLAZA 1.0 functional annotations from PLAZA server & 0.02 & 33 & & & \\
\hline Load of Gymno PLAZA 1.0 data into TOA database & 0.02 & 281 & & & \\
\hline Build of Dicots PLAZA 4.0 proteome & 0.02 & 280 & & & \\
\hline Download of Dicots PLAZA 4.0 functional annotations from PLAZA server & 0.02 & 81 & & & \\
\hline Load of Dicots PLAZA 4.0 data into TOA database & 0.02 & 1100 & & & \\
\hline Build of Monocots PLAZA 4.0 proteome & 0.02 & 234 & & & \\
\hline Download of Monocots PLAZA 4.0 functional annotations from PLAZA server & 0.02 & 48 & & & \\
\hline Load of Monocots PLAZA 4.0 data into TOA database & 0.02 & 404 & & & \\
\hline Build of NCBI RefSeq Plant proteome & 0.02 & 286 & \multirow{3}{*}{$\begin{array}{l}\text { c5.xlarge } \\
\text { (spot) }\end{array}$} & \multirow{3}{*}{4} & \multirow{3}{*}{8} \\
\hline Build of NCBI BLAST NT database for BLAST+ & 0.02 & 4843 & & & \\
\hline Build of NCBI BLAST NR database for BLAST + & 0.02 & 7900 & & & \\
\hline Build of NCBI BLAST NR database for DIAMOND & 0.04 & 8416 & r5.xlarge & 4 & 32 \\
\hline
\end{tabular}


(spot)

\begin{tabular}{llll}
\hline Download of NCBI Gene functional annotations from NCBI server & 0.02 & 7 \\
\cline { 1 - 3 } Load of NCBI Gene data into TOA database & 0.02 & 1532 \\
\cline { 1 - 3 } Download of InterPro functional annotations from InterPro server & 0.02 & 2 & 2 \\
\cline { 1 - 3 } Load of InterPro data into TOA database & 0.02 & 2 \\
\cline { 1 - 2 } Download of Gene Ontology functional annotations from Gene Ontology server & 0.02 & 1 \\
\hline Load of Gene Ontology data into TOA database & 0.02 & 3 \\
\hline
\end{tabular}




\section{Table 6(on next page)}

Tests of TOA pipeline processes performance in the functional annotation workflow.

Output size and elapsed time (E.T.) consumed by each process and type of instance, number of CPUs and RAM employed to run each process. 
1 Table 6. Tests of TOA pipeline processes performance in the functional annotation workflow. Output size and elapsed time (E.T.) consumed by each 2 process and type of instance, number of CPUs and RAM employed to run each process.

\begin{tabular}{|c|c|c|c|c|c|}
\hline Process & $\begin{array}{l}\text { Output Size } \\
\text { (MB) }\end{array}$ & E.T. (s) & Instance & vCPU & $\begin{array}{l}\text { RAM } \\
\text { (GiB) }\end{array}$ \\
\hline Uploading of reference dataset file (MonolignolsGenes.fasta) & - & - & $\begin{array}{l}\text { t3.medium } \\
\text { (spot) }\end{array}$ & 2 & 4 \\
\hline TOA nucleotide pipeline using BLAST $+->$ annotation 1 & 15.45 & 1041 & \multirow{4}{*}{$\begin{array}{l}\text { r5.xlarge } \\
\text { (spot) }\end{array}$} & \multirow{4}{*}{4} & \multirow{4}{*}{32} \\
\hline TOA nucleotide pipeline using DIAMOND -> annotation 2 & 24.11 & 166 & & & \\
\hline TOA amino acid pipeline using BLAST $+->$ annotation 3 & 32.13 & 4997 & & & \\
\hline TOA amino acid pipeline using DIAMOND -> annotation 4 & 41.41 & 7006 & & & \\
\hline Annotation merger of TOA pipelines using annotation $1 \mathrm{y}$ annotation 3 & 5.49 & 7 & \multirow{2}{*}{$\begin{array}{l}\text { t3.medium } \\
\text { (spot) }\end{array}$} & \multirow{2}{*}{2} & \multirow{2}{*}{4} \\
\hline Annotation merger of TOA pipelines using annotation $2 \mathrm{y}$ annotation 4 & 2.44 & 1 & & & \\
\hline
\end{tabular}




\section{Table 7 (on next page)}

Comparison between NGScloud2 and the Galaxy framework.

Galaxy employs several hardware infrastructure types (Galaxy cluster, Jetstream,

Stampede2, PSC Bridges). Galaxy Data Source: https://galaxyproject.org/main/ 
1 Table 7. Comparison between NGScloud2 and the Galaxy framework. Galaxy employs several hardware infrastructure types (Galaxy cluster, Jetstream,

2 Stampede2, PSC Bridges).

\begin{tabular}{|c|c|c|}
\hline Feature & NGScloud2 & Galaxy (https://usegalaxy.org/) \\
\hline Expense & According to use & Free \\
\hline Availability & Immediate & $\begin{array}{l}\text { Galaxy cluster: short/moderade wait } \\
\text { Jetstream: short/moderade wait } \\
\text { Stampede2 (normal): moderate/long wait } \\
\text { Stampede2 (SKX): long/very long wait } \\
\text { PSC Bridges: moderate/long wait }\end{array}$ \\
\hline Maximum storage size (GB) & Unlimited & 250 GB for registered users \\
\hline Data privacy & Strong & Moderate \\
\hline Maximum walltime (hours) & Unlimited & $\begin{array}{l}\text { Galaxy cluster: } 36 \\
\text { Jetstream: } 36 \\
\text { Stampede2 (normal): } 48 \\
\text { Stampede2 (SKX): } 48 \\
\text { PSC Bridges: } 24-96\end{array}$ \\
\hline Maximum CPUs number & 96 & $\begin{array}{l}\text { Galaxy cluster: } 6 \\
\text { Jetstream: } 10 \\
\text { Stampede2 (normal): } 64 \\
\text { Stampede2 (SKX): } 48 \\
\text { PSC Bridges: } 5-20 \\
\end{array}$ \\
\hline Maximum RAM size & 768 & $\begin{array}{l}\text { Galaxy cluster: } 30 \\
\text { Jetstream: } 30 \\
\text { Stampede2 (normal): } 96 \\
\text { Stampede2 (SKX): } 192 \\
\text { PSC Bridges: } 240-960 \\
\end{array}$ \\
\hline Maximum concurrent jobs & Unlimited & 6 for registered users \\
\hline De novo RNA workflow & $\begin{array}{l}\text { Specific \& } \\
\text { flexible }\end{array}$ & User-designed \\
\hline Reference-based RNA-seq workflow & $\begin{array}{l}\text { Specific \& } \\
\text { flexible }\end{array}$ & User-designed \\
\hline RAD-seq workflow & $\begin{array}{l}\text { Specific \& } \\
\text { flexible }\end{array}$ & No \\
\hline Functional annotation workflow & $\begin{array}{l}\text { Specific \& } \\
\text { flexible }\end{array}$ & User-designed \\
\hline Other workflows & No & User-designed \\
\hline
\end{tabular}




\section{Figure 1}

Technical improvements of NGScloud2.

Hardware infrastructure in AWS's cloud can be setup using a cluster mode with only the previous generation on-demand instances or a native mode that allows configuring new generation on-demand/spot instances. Apps, reads, datasets, references and results can be stored using multiple volumes or a single storage volume containing specific working directories. All the cloud configuration processes are controlled from the NGScloud2 GUI installed on a local computer. 


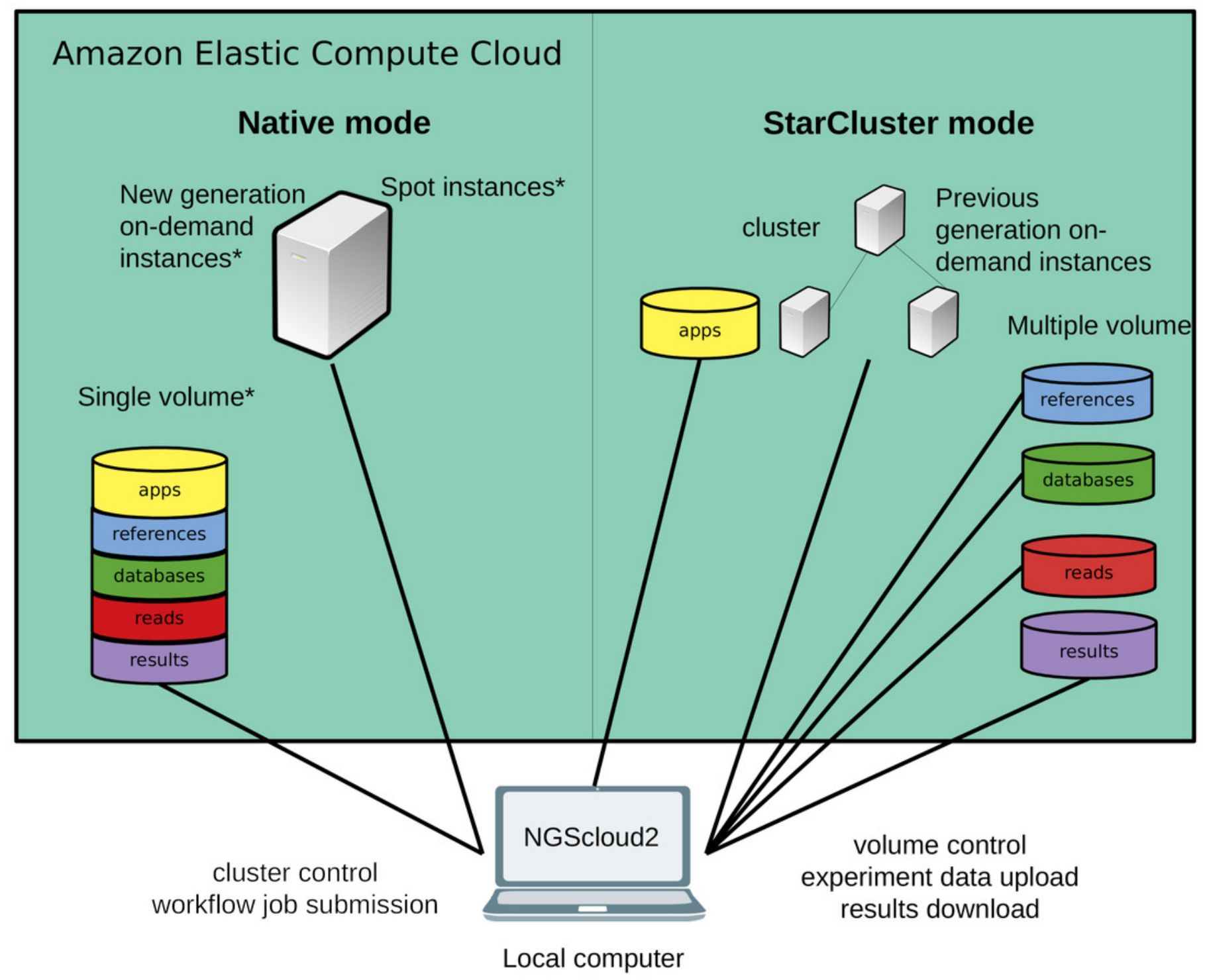




\section{Figure 2}

De novo RNAseq workflow in NGScloud2.

The workflow has the possibility to select several applications to perform each step of the analysis: read pre-processing, de novo transcriptome assembly, transcriptome filtering and quality assessment, read mapping, quantitation, variant calling and transcript annotation. The selection of applications for each step in the workflow and the parameter configuration are controlled from the NGScloud2 GUI installed on a local computer. 


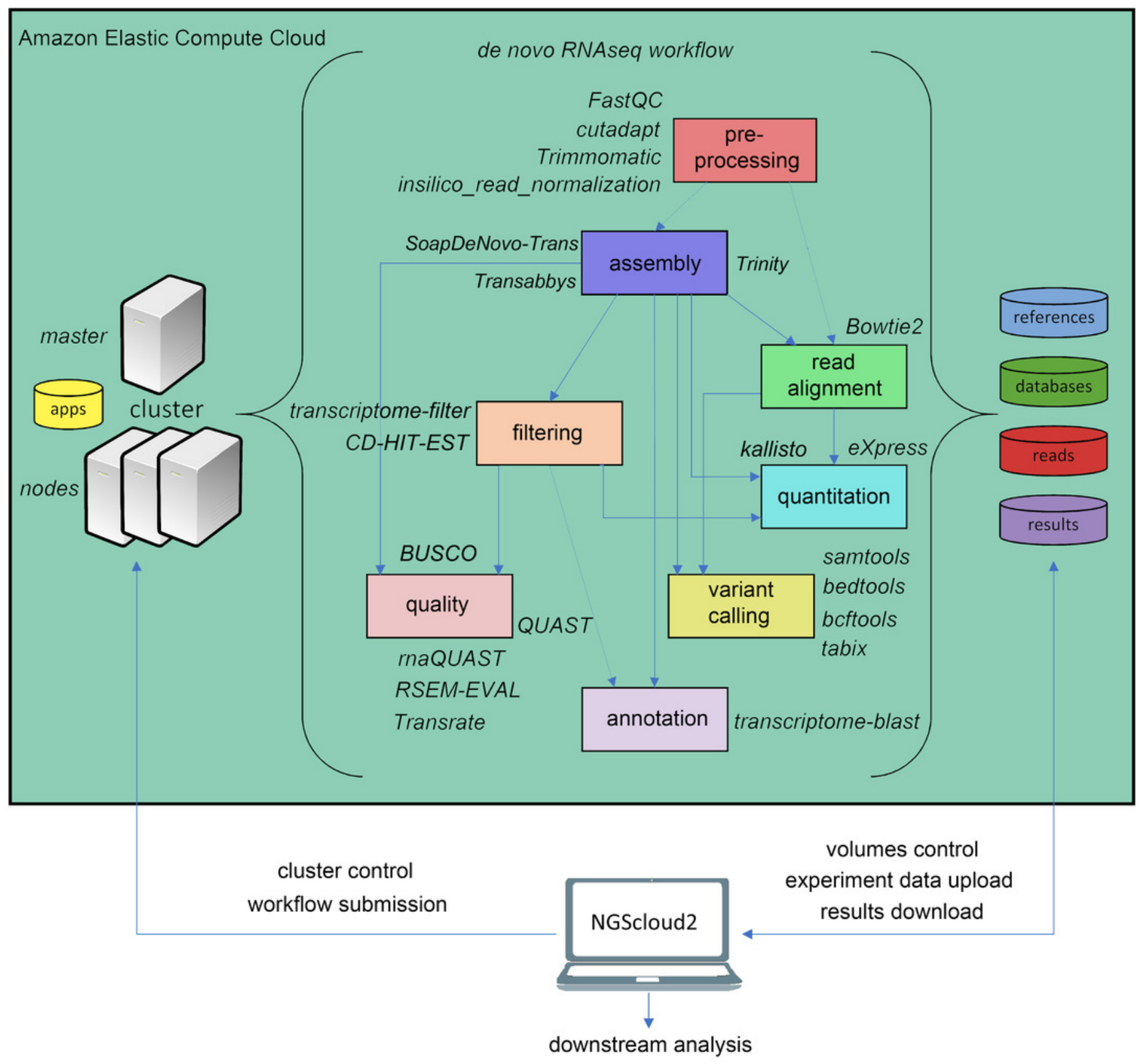




\section{Figure 3}

Reference-based RNAseq workflow in NGScloud2.

The workflow has the possibility to select several applications to perform each step of the analysis: read pre-processing, read alignment, transcriptome assembly, transcript alignment, variant calling quantitation and differential expression. The selection of applications for each step in the workflow and the parameter configuration are controlled from the NGScloud2 GUI installed on a local computer.

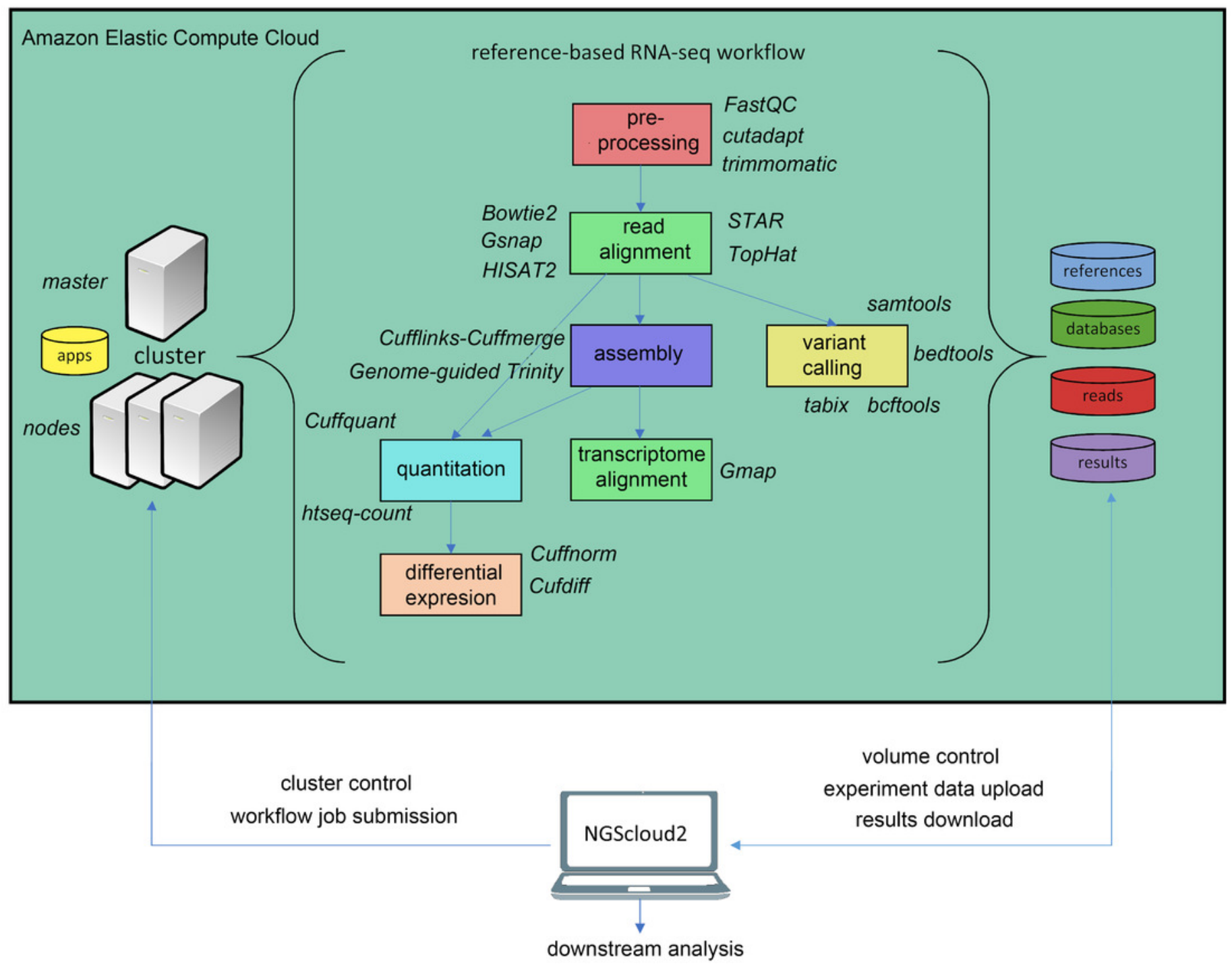




\section{Figure 4}

RADseq workflows in NGScloud2.

The reference-based RAD-seq workflow has the possibility to select several applications to perform each step of the analysis: RAD-seq experiment design, read pre-processing, pseudoassembly, read alignment, and variant calling. Alternatively, NGScloud2 allows to run the full iPyrad pipeline on the cloud. The selection of applications for each step in the workflow and the parameter configuration are controlled from the NGScloud2 GUI installed on a local computer. 


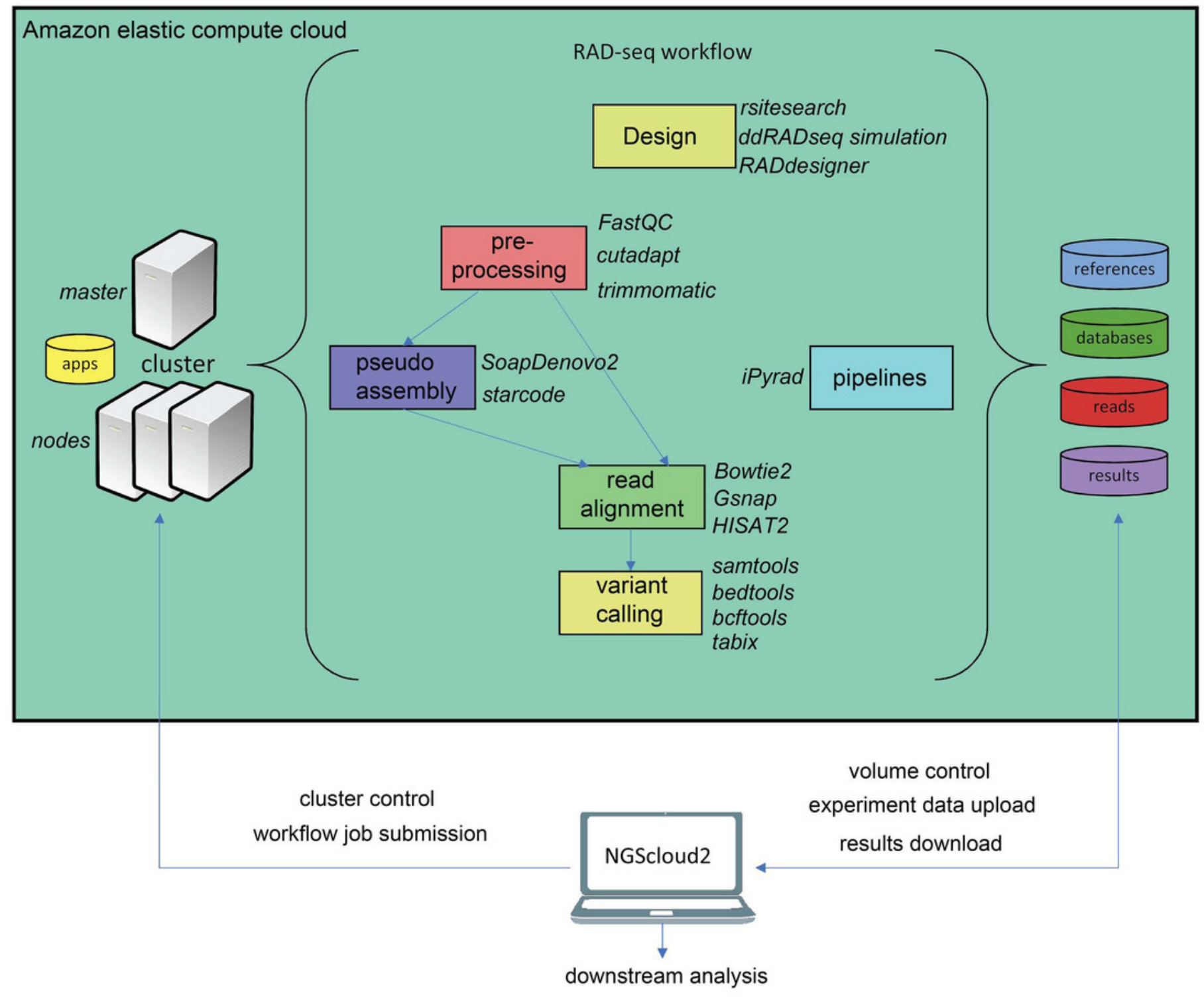




\section{Figure 5}

Functional annotation workflow in NGScloud2.

The functional annotation workflow allows running TOA (Taxonomy-oriented annotation) in AWS's cloud. Pipelines to run functional annotation are executed in the cloud. The databases of reference and the order they are explored can be configured. Query sequences (transcripts or DNA fragments) are aligned to TOA database using either BLAST+ or DIAMOND, and functional annotation information from several ontology systems (GO, KEGG, E.C., MetaCYC) is extracted to annotation report files that can be merged and used to build statistic files and ready-to-publish figures. The load of external genomic databases into TOA database, the selection of pipelines and the parameter configuration of TOA runs are controlled from the NGScloud2 GUI installed on a local computer. 


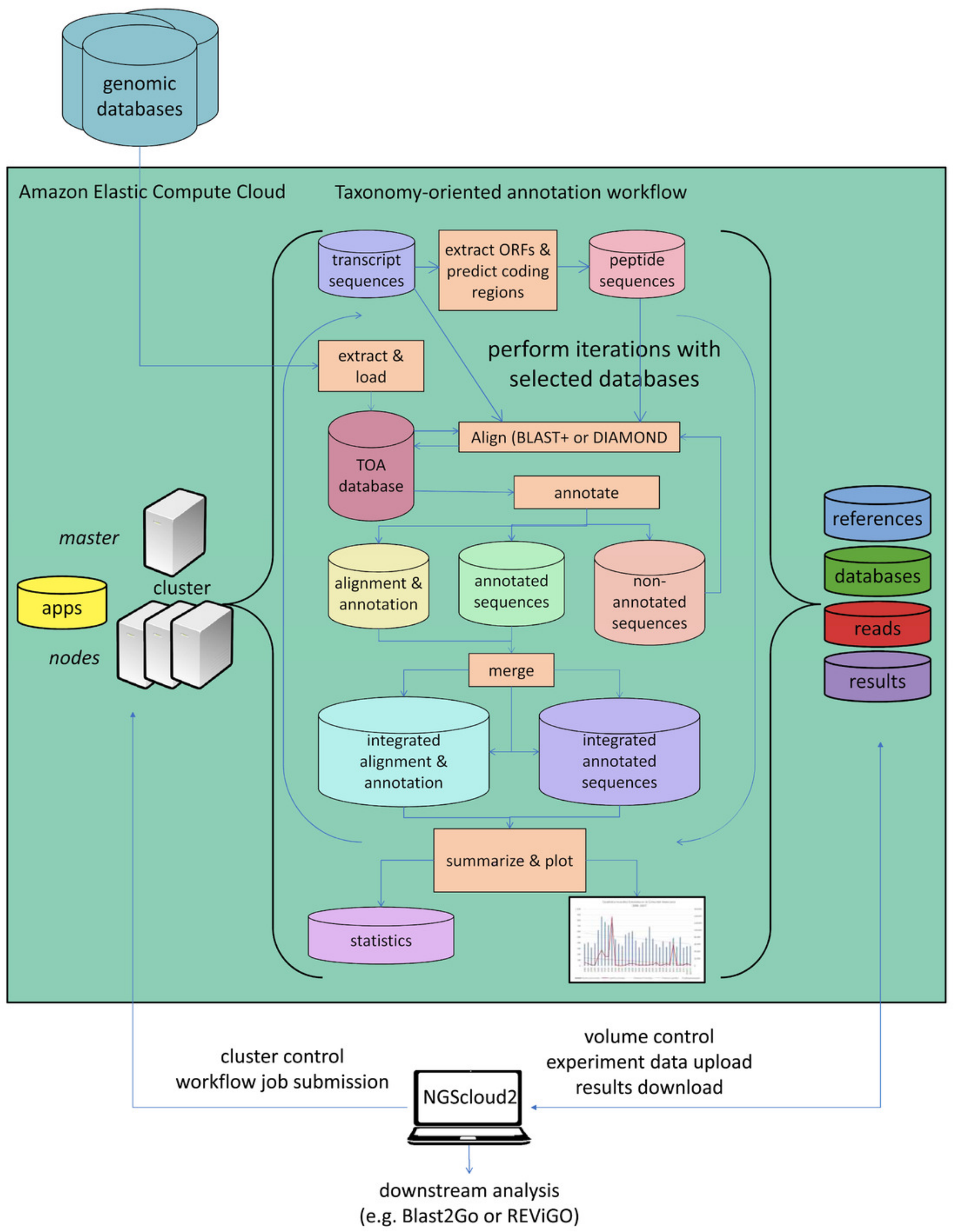

